VOL. 1, NRO. 1

\section{MAYO - OCTUBRE 2021}

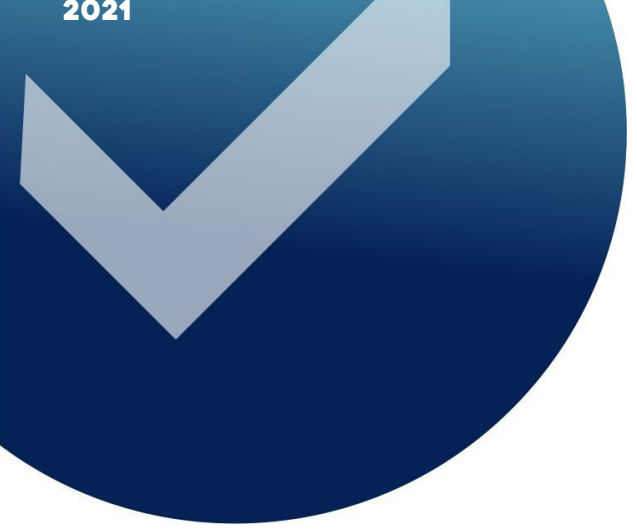

\title{
ASEGURAMIENTO RAZONABLE DE LA INFORMACIÓN CONTABLE PROSPECTIVA
}

\section{REASONABLE ASSURANCE OF PROSPECTIVE FINANCIAL INFORMATION}

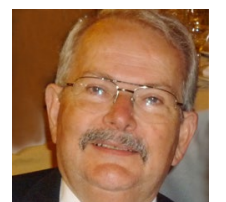

Gayetano A. V. Mora

Ex profesor titular regular de Auditoría en la Facultad de Ciencias Económicas de la Universidad Nacional del Centro de la Provincia de Buenos Aires. Argentina. mora.cayetano@gmail.com

\section{RESUMEN}

El presente trabajo tiene como objetivo reflexionar acerca de la posibilidad de emitir un informe de seguridad razonable sobre información contable prospectiva, fundamentalmente en la alternativa de un pronóstico.

\section{ABSTRACT}

This paper aims at reflecting on the possibility of issuing a reasonable assurance report on prospective financial information, fundamentally in the form of a forecast.

\section{KEYWORDS}

reasonable assurance, forecasts, assurance engagement, reasonable hypothesis.

ASEGURAMIENTO RAZONABLE DE LA INFORMACIÓN CONTABLE PROSPECTIVA

\section{AUTOR:}

Cayetano A. V. Mora
RECIBIDO:

7 de diciembre, 2020

APROBADO:

24 de marzo, 2021

\section{AUDITAR}

PRIMERA REVISTA ARGENTINA

EXCLUSIVA SOBRE AUDITORÍA
DOI: https://doi.org/10.24215/27188647e003 CÓDIGO JEL: M42

ISSN: 27-18-8647

http://revistas.unlp.edu.ar/auditar

\section{ENTIDAD EDITORA:}

Instituto de Investigaciones y Estudios

Contables, Facultad de Ciencias Económicas, Universidad Nacional de La Plata 


\section{INTRODUCCIÓN}

Las normas internacionales sobre encargos de aseguramiento diferentes de la auditoría y la revisión de información financiera histórica (NIEA 3000) así como las normas argentinas de la Resolución Técnica № 37 (RT 37) tratan lo relativo al examen de la información financiera prospectiva en la NIEA 3400 y en el Capítulo V.B. respectivamente.

Ambos textos, por otra parte, incluyen una afirmación que se habrá de destacar seguidamente por cuanto constituye el objetivo central de este trabajo.

Dicha afirmación está incluida bajo el título de "Grado de seguridad proporcionado por el auditor con respecto a información financiera prospectiva", donde, en el apartado 8, la NIEA 3400 explica que la información financiera prospectiva se refiere a hechos y acciones que todavía no han tenido lugar y que podría resultar que no ocurran nunca. Pone de resalto que la evidencia para sustentar las hipótesis en las que se basa la información financiera (o contable, como es habitual referirse a ella en Argentina) está por lo general orientada al futuro y que, por lo tanto, es de carácter especulativo, afirmación que, sin hesitar, el autor comparte.

Pero el tema al que se alude se encuentra en el final del apartado 9 de la NIEA antes citada, cuando, luego de afirmar que "al auditor le puede resultar difícil obtener un grado de satisfacción suficiente como para expresar una opinión positiva de que las hipótesis están libres de incorrección material", y normalmente solo proporciona un grado de seguridad limitado, el mencionado párrafo finaliza diciendo lo siguiente: "Sin embargo, si a juicio del auditor se ha obtenido un grado de satisfacción adecuado, no se excluye la posibilidad de que exprese seguridad de forma positiva con respecto a las hipótesis".

\section{LA INFORMACIÓN FINANCIERA PROSPECTIVA EN LAS NORMAS}

La norma argentina que trata el tema del aseguramiento de la información prospectiva está basada en la norma internacional y por tal motivo se hará seguidamente una breve des- cripción de la disposición que regula todos aquellos encargos que son diferentes de la auditoría o la revisión de información financiera histórica.

La disposición señalada es la NIEA 3000 (Revisada) y está destinada a contemplar diversos encargos que, en sustancia, son similares 0 iguales a una auditoría 0 a una revisión, pero donde la materia objeto de la tarea es diferente de la información financiera histórica.

Va de suyo que al decir "histórico" la norma no establece una diferencia con una información financiera ajustada por inflación, sino que apunta a comprender genéricamente otros asuntos, temas o materias. El término "histórico" refiere a información de hechos pasados y no a la moneda en que esa información se expresa.

En la situación bajo examen, diferencia la información que surge de un juego de estados contables, basada en los hechos del pasado y sus repercusiones futuras, de aquella que trata sobre estimaciones acerca de lo que podría ocurrir en el futuro, tal como son los estados prospectivos.

Esta NIEA 3000 tiene gran importancia porque, como se ha afirmado, está destinada a regular una enorme cantidad de servicios donde el contador agrega credibilidad, es decir que otorga un cierto aseguramiento, sobre muchas materias 0 asuntos diferentes que podrían ir desde los ya comentados estados prospectivos hasta el relativamente nuevo balance social, aunque solo para la parte destinada a la memoria de sustentabilidad. En el caso del balance social todo lo correspondiente al estado de valor económico generado y distribuido se guiará por las normas contables argentinas 0 por las normas internacionales de información financiera.

La identificación de algunos de los múltiples servicios que podrían prestarse utilizando dicha NIEA se detallan seguidamente con el único objetivo de advertir, por un lado, la enorme cantidad de encargos que los contadores podrían realizar si contaran con los conocimientos, personal y experticia requeridos y, por el otro, la importancia que tiene para el ejercicio profesional:

A- Actualmente con normas específicas

NIEA 3400. Información financiera prospectiva

NIEA 3402. Sobre los controles de una organización de servicios 
NIEA 3410. Aseguramiento sobre declaraciones de gases de efecto invernadero

NIEA 3420. Aseguramiento para informar sobre la compilación de información contable proforma incluida en un folleto

B- Sin normas específicas pero incluidos dentro de la NIEA 3000 (y comprendidos en la RT 37)

- Conclusión sobre cumplimiento de convenios contractuales

- Confiabilidad de sistemas, tales como sistemas de control interno, sistemas de información ambiental, sistemas destinados a la obtención y capacitación de recursos humanos, comportamiento del gobierno de las sociedades (corporate governance)

Informes sobre datos estadísticos

Informes sobre cumplimiento de normas

Confiabilidad del comercio electrónico

Informes sobre medición del rendimiento

Capital intelectual

Responsabilidad social empresaria

Adecuación del sistema contable a las necesidades de un ente

- Cumplimiento de presupuestos

- Aseguramiento sobre la determinación de costos de producción

- Indicadores de rendimiento, productividad, reclamos y otros

- Estructuras de costos de producción

Ingreso de personas a una página web

Muchísimos otros asuntos o materias

Regresando ahora a la NIEA 3400 sobre información financiera prospectiva, si un lector se preguntara qué es lo que está diciendo la norma cuando afirma que no se excluye la posibilidad de que el contador exprese seguridad de forma positiva con respecto a las hipótesis, debería concluir que:

no se refiere a la posibilidad de que los resultados de la exploración de posibilidades futuras basada en indicios presentes coincida con los sucesos que habrán de ocurrir, y

no afirma que en ese caso las expectativas, los supues- tos y las hipótesis concordarán con lo que efectivamente habrá de suceder, con lo que una información contable 0 no contable esperada resultaría igual o muy semejante a la real, sino a algo, también importante pero sumamente diferente.

No se está aludiendo a la razonabilidad del resultado de la prospección, sino a la razonabilidad de los supuestos, hipótesis y expectativas utilizados en su preparación. Sería como diferenciar entre medios y fines.

En la información proyectada, el fin es lograr que la proyección resulte una adecuada aproximación de lo que habrá de suceder. Para ello el preparador necesitaría contar con supuestos, hipótesis y expectativas "adecuadas" si bastase con un aseguramiento limitado que solo afirme que no son irrazonables 0 en los casos de aseguramiento razonables que ellas son "razonables", para permitir que el contador pueda considerarlas como tales.

El camino por seguir, los procedimientos a aplicar y las evidencias a recoger no resultarán fáciles pero tampoco imposibles. Casi con seguridad, el riesgo del contador se incrementará, pero, como decía nuestro querido (e inmenso) Mario Wainstein hace más de quince años, el contador debe estar dispuesto a correr riesgos si pretende prestigiar a la profesión.

Para introducir algunos conceptos importantes y facilitar la interpretación de lo que se manifestará, la NIEA 3400 en la página 229 establece una distinción entre pronóstico y proyección y los define de la siguiente manera:

"Pronóstico" significa información financiera prospectiva preparada sobre la base de hipótesis acerca de hechos futuros que la dirección espera que ocurran y medidas que la dirección espera tomar en la fecha a la que se refiere la información que se prepara (la mejor estimación).

"Proyección, extrapolación" significa información financiera prospectiva preparada sobre la base de:

a- Casos hipotéticos sobre hechos futuros y actuaciones de la dirección que no necesariamente se espera que se produzcan, 0

b- una combinación de las mejores estimaciones y de un caso hipotético. 


\section{REQUERIMIENTOS PARA LA REALIZACIÓN DE ESTE ENCARGO DE ASEGURAMIENTO}

Los objetivos perseguidos por el contador en un encargo de aseguramiento de este tipo son los siguientes:

obtener seguridad razonable o seguridad limitada según lo comprometido con el comitente acerca de que el asunto 0 materia objeto de análisis está libre de incorrección material, y

expresar una conclusión sobre el resultado de su tarea, comunicando, en su caso, los aspectos requeridos por la norma respectiva.

Existen dos tipos de encargos de aseguramiento descriptos en el Marco internacional de encargos de aseguramiento e incluidos también en la NIEA 3000 y ellos son:

encargo de informe directo, $y$

encargo de constatación

A continuación, se realizará una descripción de las principales diferencias existentes entre ellos.

En un encargo de informe directo, el contador mide o evalúa el asunto o la materia objeto de análisis sobre la base de ciertos criterios y presenta la información sobre el asunto 0 materia integrando el informe de aseguramiento o como un anexo a dicho informe.

Como ejemplo podría darse que la dirección de una entidad contrate a un contador para que realice una evaluación del control interno (materia objeto de análisis) mediante la aplicación de ciertos criterios (por ejemplo el Informe COSO) y emita un informe de aseguramiento donde consten las conclusiones del contador.

En un encargo de constatación (anteriormente denominado "sobre afirmaciones"), una parte distinta al contador es la que mide 0 evalúa el asunto o la materia, pudiendo ser la misma parte responsable del asunto o materia evaluada u otra parte. Un simple ejemplo servirá para entender mejor las diferencias.

Si en una entidad el gerente de producción quisiera obtener un informe de aseguramiento de contador sobre las prácticas de mantenimiento que se aplican en su departamento, le podría pedir al responsable de mantenimiento que "mida 0 evalúe" las prácticas y entregue el informe que produzca al contador para que este realice su tarea y emita un informe sobre la evaluación que ha realizado el responsable de mantenimiento.

En este caso, se ha agregado una figura más y en lugar de existir la parte responsable (gerente de producción), el contador y los usuarios como ocurriría en el caso de un informe directo, se tendría a la parte responsable (el gerente de producción), el evaluador o medidor (el responsable de mantenimiento), el contador y los usuarios.

En la Tabla 1 se presenta en forma comparativa un breve resumen de sus aspectos principales.

\section{Tabla 1}

\section{Diferencias entre un informe directo y uno de constatación}

\begin{tabular}{|c|c|}
\hline INFORME DIRECTO & CONSTATACIÓN \\
\hline $\begin{array}{l}\text { El contador es el } \\
\text { medidor } 0 \text { evaluador }\end{array}$ & $\begin{array}{l}\text { Parte distinta del } \\
\text { contador es el medidor } \\
\text { o evaluador }\end{array}$ \\
\hline $\begin{array}{l}\text { Sobre la base de ciertos } \\
\text { criterios }\end{array}$ & $\begin{array}{l}\text { Sobre la base de ciertos } \\
\text { criterios }\end{array}$ \\
\hline $\begin{array}{l}\text { Presenta la información } \\
\text { sobre la materia objeto } \\
\text { de análisis como parte } \\
\text { del informe de } \\
\text { aseguramiento o como } \\
\text { anexo a aquél }\end{array}$ & $\begin{array}{l}\text { Obtiene como resultado } \\
\text { la información sobre la } \\
\text { materia objeto de } \\
\text { análisis }\end{array}$ \\
\hline $\begin{array}{l}\text { La conclusión del } \\
\text { contador se refiere al } \\
\text { resultado que ha } \\
\text { obtenido de la medida } \\
\text { o evaluación de la } \\
\text { materia subyacente } \\
\text { objeto de análisis } \\
\text { sobre la base de ciertos } \\
\text { criterios }\end{array}$ & $\begin{array}{l}\text { La conclusión del } \\
\text { contador trata de si la } \\
\text { información sobre la } \\
\text { materia objeto de } \\
\text { análisis está libre de } \\
\text { incorrección material }\end{array}$ \\
\hline
\end{tabular}

Fuente: elaboración propia

En el caso de la información prospectiva solo será posible realizar un encargo de constatación por cuanto las hipótesis, premisas y supuestos deberán ser establecidos por la entidad, ya que representan la visión de su dirección y porque, 
en caso contrario, se daría la anómala situación de que el contador emita un informe de aseguramiento sobre su propia gestión.

\section{EL CONTADOR TIENE PREVISTO EMITIR UNA CONCLUSIÓN SOBRE LA RAZONABILIDAD DE LOS SUPUESTOS E HIPÓTESIS}

En esta situación, independientemente de que algunas tareas a llevar a cabo serán identificadas más adelante, por encontrarse todas las actividades referidas a la "medición o evaluación" de la información prospectiva, bajo la dirección y las decisiones del contador, este no se encontraría en principio con problemas especiales, más allá de aquellos que puedan derivarse de los elementos de juicio que pueda obtener sobre:

la determinación que ha realizado la parte responsable de los supuestos que ha establecido,

las estimaciones que han efectuado,

las propias evidencias utilizadas, $y$

los razonamientos que haya efectuado hasta transformarlas en "su visión" de lo que espera que ocurra.

En la posibilidad de que se realice bajo el formato de una proyección, muchas de estas conjeturas quedarán dejadas de lado toda vez que, si se han definido bajo la idea de "qué pasaría si", no se hace una manifestación de expectativa razonable, sino una exteriorización de un escenario tal vez posible pero no necesariamente probable.

En la proyección, aparte de establecer solo un escenario, el contador tendrá en cuenta todas las implicaciones y consecuencias de cada uno de los supuestos adoptados de forma tal que, sin necesitar evidencias que corroboren las hipótesis, estas deben ser consistentes con el objetivo de la proyección y ser realistas, es decir, necesitan contar con argumentaciones sólidas y, en su caso, respaldadas por expertos.

Por ejemplo, si una entidad prepara una proyección presentando como escenario un crecimiento del producto bruto interno de un país en un cierto porcentaje y el auditor necesitase obtener elementos de juicio que lo respalden, además de Ios análisis que realice sobre lo ocurrido en años anteriores y de las proyecciones que realicen analistas, podría recurrir a un licenciado en Economía para verificar que los supuestos adoptados son posibles, es decir, si son realistas.

Finalmente, si la alternativa elegida por el comitente fuese una combinación de las mejores estimaciones y de un caso hipotético, el contador deberá realizar por una parte, una evaluación de los supuestos mejor estimados teniendo en cuenta los conceptos antes señalados y, luego, incorporar la situación originada por el "qué pasaría si".

Un ejemplo puede ayudar a interpretar mejor estas afirmaciones. Si se tratara de un estado de información contable prospectiva a tres años, donde la entidad está dispuesta a solicitar financiación bancaria para automatizar una parte de su planta industrial, un posible camino a seguir sería el siguiente:

realizar la mejor estimación posible de las diferentes variables (económicas, financieras, laborales, de mercado y otras) con el pensamiento puesto en lo que la dirección piensa que puede ocurrir durante ese período y de las acciones que emprenderá como consecuencia, y posteriormente incorporar a la prospección anterior qué es lo que podría suceder si la entidad:

- consiguiese la financiación bancaria solicitada

- con el costo financiero estimado

- comprara, instalara y comenzara a producir con la parte nueva automatizada en el tiempo previsto producir en consecuencia dos estados proyectados, uno de ellos que siguiera los hechos estimados, sin tener en cuenta la nueva inversión, y otro que reflejara los cambios que se producirían como consecuencia de la adquisición de los elementos necesarios para automatizar una parte de la planta industrial, en términos de erogaciones $\mathrm{y}$ de ingresos.

\section{PRECISIONES NECESARIAS}

Como el objetivo del trabajo es discurrir sobre la posibilidad de emitir una conclusión sobre la razonabilidad de la información prospectiva, ¿cómo se podría llegar a la conclusión de que un supuesto es razonable?

Como se ha mencionado anteriormente, la NIEA 3400 expresa en el apartado 9 que "al auditor le puede resultar difícil ob- 
tener un grado de satisfacción suficiente como para expresar una opinión positiva de que las hipótesis están libres de incorrección material”. De esta afirmación surge con claridad que la tarea del contador consistirá en verificar que el supuesto 0 la premisa se ha establecido sin incorrecciones significativas y el término que habitualmente se utiliza en auditoría es que ese supuesto, premisa o hipótesis es razonable, por lo que surge la necesidad de intentar conceptualizarlo.

"Razonable" y "razonablemente" aparecen repetidamente en la norma internacional de contabilidad № 1 cuando en el apartado 7 se refiere a la información que podría razonablemente esperarse, a "si los usuarios tienen un conocimiento razonable de las actividades económicas", cuando señala a la medición a "valor razonable" en el mismo apartado o cuando titula al apartado 15 "presentación razonable y cumplimiento de las NIIF", pero no define lo que se entiende por razonable. Por su parte, en las normas internacionales de auditoría, de revisión, de otros encargos de aseguramiento y de servicios relacionados, se utiliza el término muchas veces en "valor razonable", "seguridad razonable" y "base razonable", en "posibilidad razonable" y en "estimaciones contables razonables", entre otras.

Sin embargo, por los diferentes usos a los que se asocia este término, podría decirse que puede significar "seguridad alta pero no absoluta" en la expresión "seguridad razonable", aunque cambia de algún modo cuando se expresa "alcanzar juicios razonables", "la necesidad de que la auditoría se realice en un plazo de tiempo y con un costo razonable" o "el auditor determinará si existe una justificación razonable para ello".

Con la necesidad de encontrar términos sustitutos de razonable, se podrá usar el antiguo "aproximación a la realidad" para algunas afirmaciones, "cercano al importe correcto" para otras, "adecuado, conforme a razón, proporcionado 0 no exagerado" cuando la norma internacional para encargos de revisión establece en el apartado A72 que "es razonable que el profesional acepte dicha redacción..." y "alta pero no absoluta" cuando se trata de seguridad razonable.

Así que, como conclusión, lo que el contador debería obtener es una alta pero no absoluta seguridad de que las premisas utilizadas, que harían que la información prospectiva se aproxime a la realidad, son apropiadas porque disminuyen el riesgo de que contengan incorrecciones a un nivel suficientemente bajo como para permitirle afirmar que "en su opinión esas hipótesis son razonables".

Como se puede advertir, en ningún momento se ha hecho mención de que los resultados obtenidos ex post, es decir, luego de ocurridos los acontecimientos sobre los cuales se hicieron las hipótesis, premisas o supuestos, se hayan acercado a la realidad, aunque sería de esperar que en muchos casos resulten cercanos si los acontecimientos previstos se desarrollaron en la forma en que se habían supuesto.

En países como la Argentina, donde la situación económica varía con suma frecuencia sorprendiendo a nacionales y extranjeros, donde a ciertos períodos de estabilidad cambiaria le siguen períodos de grandes convulsiones inesperadas, la posibilidad de que las estimaciones resulten acertadas, es decir, cercanas a la realidad, no depende en muchas oportunidades de la capacidad o experticia del evaluador sino del momento histórico en que se esté llevando a cabo la tarea.

\section{TAREAS POR REALIZAR EN BÚSQUEDA DE EVIDENCIAS}

Como el presente trabajo no está destinado a servir como doctrina sobre información prospectiva por estar perfectamente cubierta por prestigiosos autores, solo se tratará de identificar algunos supuestos o hipótesis que suelen utilizarse y la forma de buscar suficientes evidencias que permitan, posteriormente, emitir una conclusión redactada en forma positiva, es decir, del tipo "En mi opinión..." en remplazo de la más común "Nada llamó mi atención que me haga pensar que...".

Para ser más precisos, se supone que una empresa ha preparado un juego de estados de información financiera prospectiva en la alternativa denominada pronóstico.

La propia norma reconoce que en función de los tipos de evidencia disponible para la evaluación de los supuestos muy probablemente al auditor le puede resultar difícil obtener un grado de satisfacción que le permita afirmar que los supuestos están libres de incorrección material. Por ese motivo, 
toda la norma está redactada partiendo de la base de que solo se proporcionará un grado de seguridad moderado muchas veces mal denominado "certeza negativa".

Si bien el marco internacional no hace referencia a seguridad moderada y sí trata a los encargos de seguridad limitada, debe entenderse que la seguridad moderada se corresponde con seguridad limitada.

La expresión grado de seguridad moderado se contrapone a grado de seguridad alto y cumplen la misma función que seguridad razonable (alta pero no absoluta) y seguridad limitada (significativa como para incrementar la confianza de los usuarios pero por debajo de la seguridad razonable).

Pero como lo que busca este trabajo es avanzar hacia la seguridad alta pero no absoluta, hacia la seguridad razonable, se deberían sentar algunas bases que abran el camino en pos de ese fin.

En primer lugar, el contador debería obtener un conocimiento acerca de si el cliente tiene experiencia en la preparación de presupuestos y proyecciones y, en ese caso, cuál ha sido el resultado de ellos en el pasado.

En ese caso será importante conocer no solo las diferencias numéricas determinadas entre las estimaciones y lo efectivamente ocurrido, sino los argumentos presentados para identificar las causas de las diferencias.

En algún momento posterior, el contador solicitará información relevante sobre los supuestos que ha considerado la administración y su justificación, tratando de concentrar su tarea en aquellos que son más sensibles, es decir, los supuestos en los que una pequeña variación genera una modificación significativa en el resultado.

Si la entidad fuera una empresa industrial que utiliza algunos insumos importados, la inflación así como la futura cotización de la moneda extranjera seguramente serán elementos para tener en cuenta particularmente por las variaciones en las materias primas importadas, en el costo de la mano de obra así como de los demás insumos que se adquieren en el país y en el extranjero, los que con frecuencia son difíciles de estimar.

Ahora bien, si el aseguramiento contratado al auditor fuese de seguridad limitada, donde solo debe afirmar que los supuestos no son irrazonables, la tarea sería compleja pero dispon- dría de un margen de maniobra importante, ya que diferenciar entre razonable e irrazonable deja un espacio relativamente amplio, una distancia apreciable, un intervalo frecuentemente cómodo como para afirmar en un pronóstico "Basándome en mi examen de la evidencia que sustenta las hipótesis, no ha llegado a mi conocimiento ninguna cuestión que me lleve a pensar que dichas hipótesis no constituyen una base razonable para el pronóstico".

En cambio, muy diferente sería la cuestión si el contador tuviera como objetivo emitir un informe que dijera "Basándome en mi examen de la evidencia que sustenta las hipótesis, en mi opinión, las hipótesis constituyen una base razonable para el pronóstico. Además, en mi opinión, el pronóstico ha sido adecuadamente preparado sobre la base de las hipótesis y se presenta de acuerdo con las normas contables profesionales".

La suma de los "razonables" anteriores, la fuerza de sus "aproximaciones a la realidad", lo "adecuado y cercano a los correctos" que han sido los supuestos, están afirmando que ellos no contienen incorrecciones importantes y agregará un elemento de convicción para las decisiones que tomen los usuarios del informe.

¿Incrementa el riesgo del contador de emitir un informe inadecuado? Efectivamente, porque el espacio relativamente amplio y la distancia apreciable que puede existir entre afirmar que algo no es irrazonable a que ese mismo algo es razonable desaparecieron. Valga un ejemplo práctico como modelo.

Si se hiciera referencia a la inflación mensual de un período de tiempo cualquiera, si una estimación fuera del 2\% mensual, el 1,5 o el 2,5 no serían irrazonables, porque dependiendo del momento en que han ocurrido las variaciones, de su efecto en el índice que calcula el organismo público, de la estacionalidad de lo que se está midiendo y de muchas otras consideraciones, podría afirmarse que "no es irrazonable", pero muy probablemente podrían generar muchas repercusiones en la información que impedirían referirse a ellas como "razonables o cercanas a la realidad".

Si la presupuestación incluyera un proyecto de inversión, con alguna 0 algunas de las diferentes fuentes de capital, como serían el capital del empresario, la oferta de acciones, el en- 
deudamiento bancario, con proveedores y otros, los subsidios que se espera recibir, la entidad seguramente contará con una evaluación profunda acerca de la recuperabilidad de la inversión más la obtención de un margen de rentabilidad u otros objetivos como podrían ser, a manera de ejemplo, la reducción de impactos ambientales y sociales negativos.

La participación de licenciados en Economía en función de lo establecido por la RT 50 de la FACPCE le permitiría al contador contar con un informe del profesional donde podría apreciar la opinión de aquel acerca de si los flujos proyectados en su conjunto presentan razonablemente, en todos sus aspectos significativos, la información que debe brindar de acuerdo con las normas profesionales vigentes u otro marco de información, pudiendo ser su conclusión una opinión favorable, con salvedades, adversa 0 una abstención de opinión.

En este caso, el contador, que tiene como objetivo emitir una conclusión positiva sobre la información prospectiva y que por ello necesita saber si la información que resulta de las hipótesis o supuestos es razonable, contará con la opinión de un experto y, aunque no se trate de un experto propio, en el sentido de haber sido suya la elección, podrá realizar diversas evaluaciones en torno a la competencia, capacidad y objetividad del experto, al decir de la norma internacional de auditoría 620 "Utilización del trabajo de un experto del auditor" y que brevitatis causae no se explicitan.

No obstante, los estudios de mercado, de la organización, la construcción del flujo de fondos, las técnicas de evaluación y el análisis de riesgo que podrían formar parte del trabajo del licenciado en Economía serán de suma utilidad en la búsqueda de evidencias válidas y suficientes.

En el mismo sentido, podrían ser útiles las tareas e informes realizados por licenciados en Administración en caso de contar con un informe de un plan de negocios, siguiendo las normas de la RT 49 de la FACPCE.

\section{CONCLUSIÓN}

Siguiendo entonces el camino trazado hacia el meollo de la cuestión, la pregunta de fondo podría consistir en ¿cuál podría ser el camino por seguir para llegar a la conclusión de que un supuesto o hipótesis es razonable?

La respuesta que surge (posiblemente más fácil de esbozar que de llevar a cabo) consiste en evaluar profundamente, con base en información sólida y verificable, que los supuestos, hipótesis o premisas:

han sido adecuadamente establecidos,

fundados en estimaciones objetivas, realizadas sin sesgos, conflictos de intereses o la influencia de terceros, y con la consideración de todas (o la mayoría de) aquellas circunstancias que podrían provocar cambios significativos.

Al igual que en una auditoría de estados financieros, las evidencias que se obtendrán serán por lo general más persuasivas que conclusivas, es decir que tienen fuerza y eficacia para inducir a alguien con razones a creer o hacer algo.

De esta forma el evaluador, auditor o contador en un encargo de aseguramiento prueba de una manera que racionalmente no se pueda negar, no la exactitud aritmética de una cifra al final de un proceso o la corrección del resultado de una afirmación transcurrido un cierto tiempo, sino la razonabilidad del supuesto o de una hipótesis en el momento en que se realizó, con los elementos de juicio disponibles en ese momento. ¿Con mayor riesgo, se pregunta? La respuesta es afirmativa, pero también con la satisfacción de haber obtenido elementos de juicio para incrementar la confianza de los usuarios en la información que la entidad presenta. Lo mismo ocurre entre una auditoría 0 una revisión de estados financieros. Ambos son excelentes servicios profesionales, realizados con la misma responsabilidad, pero donde en el primero se satisfacen mejor las necesidades de los usuarios.

La conclusión, si se ha logrado la convicción requerida, podría estar redactada en los términos "Basándome en mi examen de la evidencia que sustenta las hipótesis y en mi opinión, las hipótesis constituyen una base razonable para el pronóstico que ha sido adecuadamente preparado sobre la base de ellas y se presenta de acuerdo con las normas contables profesionales".

Naturalmente entonces, al final del camino señalado, el contador habrá realizado un trabajo un tanto diferente porque su objetivo se ha alterado, que incrementa la utilidad para los usuarios, que requiere de mayores conocimientos y de enjun- 
dia superadora pero donde jerarquizará su compromiso con la profesión que ejerce.

\section{REFERENCIAS}

Federación Argentina de Consejos Profesionales de Ciencias Económicas. (2013). Resolución Técnica № 37 Normas de Auditoría, Revisión, Otros Encargos de Aseguramiento, Certificación y Servicios Relacionados.

Federación Argentina de Consejos Profesionales de Ciencias Económicas. (2018). Resolución Técnica № 49. Plan de negocios. Marco conceptual e Informe de plan de negocios.

Federación Argentina de Consejos Profesionales de Ciencias Económicas. (2019). Resolución Técnica № 50. Formulación y Evaluación de proyectos de inversión.

Federación Internacional de Contadores. (Edición 2018). Manual de Pronunciamientos Internacionales de Control de Calidad, Auditoría, Revisión, Otros Encargos de Aseguramiento y Servicios Relacionados emitido por la Junta de Normas Internacionales de Auditoría y Aseguramiento (Volumen I).

Federación Internacional de Contadores. (Edición 2018). Manual de Pronunciamientos Internacionales de Control de Calidad, Auditoría, Revisión, Otros Encargos de Aseguramiento y Servicios Relacionados emitido por la Junta de Normas Internacionales de Auditoría y Aseguramiento (Volumen II).

Junta de Normas Internacionales de Contabilidad. Norma Internacional de Contabilidad 1. Presentación de estados financieros. 